\title{
Galois Connections Between Unbalanced Structures in a Fuzzy Framework
}

\author{
Inma P. Cabrera, Pablo Cordero, Emilio Muñoz-Velasco, \\ and Manuel Ojeda-Aciego ${ }^{(凶)}$ \\ Dept. Matemática Aplicada, Universidad de Málaga, Málaga, Spain \\ \{ipcabrera, pcordero, ejmunoz, aciego\}@uma.es
}

\begin{abstract}
The construction of Galois connections between unbalanced structures has received considerable attention in the recent years. In a nutshell, the problem is to find a right adjoint of a mapping defined between sets with unbalanced structure; in this paper we survey recent results obtained in this framework, focusing specially on the fuzzy structures that have been considered so far in this context: fuzzy preposets, fuzzy preordered structures, and fuzzy T-digraphs.
\end{abstract}

Keywords: Galois connection · Computational intelligence

\section{Introduction}

The notion of Galois connection (or its sibling, adjunction) has received considerable attention since its introduction [28], and it is common to find papers dealing with them either from a practical or a theoretical point of view, see [11] for a short survey. Galois connections (both in a crisp and in a fuzzy setting) have found applications in areas such as rough set theory [12,15,33]; (fuzzy) Mathematical Morphology in which the (fuzzy) operations of erosion and dilation are known to form a Galois connection [5,22,29,30]; another important source of applications of Galois connections is within the field of Formal Concept Analysis $[7,13,16,31]$, where the concept-forming operators form either an antitone or isotone Galois connection (depending on the specific definition). Moreover, one can find applications in many other areas; for instance, Kycia [26] demonstrates how to construct a Galois connection between two systems with entropy; Brattka [6] considers a formal Galois connection in a certain lattice of representation spaces; Faul [17] uses adjunctions to study two apparently different approaches to broadcast domination of product graphs; Moraschini [27] introduces a logical and algebraic description of right adjoint functors between generalized quasi-varieties; Gibbons et al. [21] use adjunctions to elegantly explain relational algebra constructs.

Concerning the generalization to the fuzzy case of the notion of Galois connection, to the best of our knowledge, the first approach was due to Bělohlávek [3]. Later, a number of authors have considered different approaches to the so-called 
fuzzy (isotone or antitone) Galois connections; see [4,14, 18, 20, 23, 24, 32]. In [32], fuzzy Galois connections on fuzzy posets were introduced as a generalization of Bělohlávek's fuzzy Galois connection, and our approach is precisely based on this generalization.

In this paper, we survey recent results in our research line on the construction of Galois connections between sets with unbalanced structures initiated in [19], in which we attempt to characterize the existence of the right part of a Galois connection of a given mapping $f: \mathbb{A} \rightarrow B$ between sets with a different structure (it is precisely this condition of different structure that makes this problem to be outside the scope of Freyd's adjoint functor theorem). In [19], given a mapping from a crisp (pre-)ordered set $\mathbb{A}=\left(A, \leq_{A}\right)$ into an unstructured set $B$, we solved the problem of defining a suitable (pre-)ordering relation $\leq_{B}$ on $B$, for which there exists a mapping such that the pair of mappings forms an isotone Galois connection (or adjunction) between the (pre-)ordered sets $\left(A, \leq_{A}\right)$ and $\left(B, \leq_{B}\right)$.

Specifically, we consider the previous problem in different fuzzy frameworks: in Sect. 3 we focus on the case of a fuzzy preposet $\mathbb{A}=\left(A, \rho_{A}\right)$ and an unstructured $B$, see [8]; later, in Sect. 4 , the work is extended by replacing crisp equality by a fuzzy equivalence relation, therefore the problem considers a mapping between a fuzzy preordered structure $\mathbb{A}=\left(A, \approx_{A}, \rho_{A}\right)$ and a fuzzy structure $\left(B, \approx_{B}\right)$, see [9]. Finally, in Sect. 5 we aim at obtaining a notion of Galois connection whose components are, in fact, relations between fuzzy T-digraphs [10].

\section{Preliminary Definitions}

The standard notion of Galois connection is defined between two partially ordered sets. However, not all the authors consider the same definition of Galois connection and it is remarkable that the definitions are not equivalent. In fact, there are four different notions of Galois connection, the most often used being the "right Galois connection" (also known as antitone Galois connection) and the "adjunction" (also known as isotone Galois connections).

Definition 1. Let $\mathbb{A}=(A, \leq)$ and $\mathbb{B}=(B, \leq)$ be posets, $f: A \rightarrow B$ and $g: B \rightarrow$ $A$ be two mappings. The pair $(f, g)$ is called a

- Right Galois Connection between $\mathbb{A}$ and $\mathbb{B}$, denoted by $(f, g): \mathbb{A} \hookrightarrow \mathbb{B}$ if, for all $a \in A$ and $b \in B$ it holds that $a \leq g(b)$ if only if $b \leq f(a)$.

- Left Galois Connection between $\mathbb{A}$ and $\mathbb{B}$, denoted by $(f, g): \mathbb{A} \square \mathbb{B}$ if, for all $a \in A$ and $b \in B$ it holds that $g(b) \leq a$ if only if $f(a) \leq b$.

- Adjunction between $\mathbb{A}$ and $\mathbb{B}$, denoted by $(f, g): \mathbb{A} \leftrightharpoons \mathbb{B}$ if, for all $a \in A$ and $b \in B$ it holds that $a \leq g(b)$ if only if $f(a) \leq b$.

- Co-Adjunction between $\mathbb{A}$ and $\mathbb{B}$, denoted $\bar{b} y(f, g): \mathbb{A} \rightleftharpoons \mathbb{B}$ if, for all $a \in A$ and $b \in B$ it holds that $g(b) \leq a$ if only if $b \leq f(a)$.

It is noteworthy that this definition is also compatible with the case of $\mathbb{A}=$ $(A, \leq)$ and $\mathbb{B}=(B, \leq)$ being preordered sets.

Taking into account the dual order, $\mathbb{A}^{\partial}=(A, \geq)$, it is not difficult to check that the following conditions are equivalent: 
1. $(f, g): \mathbb{A} \sqcup \mathbb{B}$.

2. $(f, g): \mathbb{A}^{\partial} \rightarrow \mathbb{B}^{\partial}$.
3. $(f, g): \mathbb{A} \leftrightharpoons \mathbb{B}^{\partial}$.

4. $(f, g): \mathbb{A}^{\partial} \rightleftharpoons \mathbb{B}$.

It is worth mentioning that all the results can be stated both in terms of Galois connection or adjunctions, and either in terms of the existence and construction of right adjoints (or residual mappings, namely, the component $g$ of the pair) or the existence and construction of left adjoints (or residuated mappings).

\section{Galois Connections in the Fuzzy Case}

As usual, we will consider a complete residuated lattice $\mathbb{L}=(L, \leq, \top, \perp, \otimes, \Rightarrow)$ as the underlying structure for considering the generalization to a fuzzy framework; supremum and infimum will be denoted by $\vee$ and $\wedge$, respectively.

An $\mathbb{L}$-fuzzy set is a mapping from the universe set, say $X$, to the lattice $L$, i.e. $X: U \rightarrow L$, where $X(u)$ means the degree in which $u$ belongs to $X$. We will denote $L^{A}$ to refer to the set of all mappings from $A$ to $L$.

Given $X$ and $Y$ two $\mathbb{L}$-fuzzy sets, $X$ is said to be included in $Y$, denoted as $X \subseteq Y$, if $X(u) \leq Y(u)$ for all $u \in U$. The subsethood degree $S(X, Y)$, by which $X$ is a subset of $Y$, is defined by $S(X, Y)=\bigwedge_{u \in U}(X(u) \Rightarrow Y(u))$.

The first notion of fuzzy Galois connection was given by Bělohlávek, and it can be rewritten as follows:

Definition 2 ([3]). An (ㄴ-)fuzzy Galois connection between $A$ and $B$ is a pair of mappings $f: L^{A} \rightarrow L^{B}$ and $g: L^{B} \rightarrow L^{A}$ such that, for all $X \in L^{A}$ and $Y \in L^{B}$ it holds that $S(X, g(Y))=S(Y, f(X))$.

An $\mathbb{L}$-fuzzy binary relation on $U$ is an $\mathbb{L}$-fuzzy subset of $U \times U$, that is $\rho_{U}: U \times U \rightarrow L$, and it is said to be:

- Reflexive if $\rho_{U}(a, a)=\top$ for all $a \in U$.

- $\otimes$-Transitive if $\rho_{U}(a, b) \otimes \rho_{U}(b, c) \leq \rho_{U}(a, c)$ for all $a, b, c \in U$.

- Symmetric if $\rho_{U}(a, b)=\rho_{U}(b, a)$ for all $a, b \in U$.

- Antisymmetric if $\rho_{U}(a, b)=\rho_{U}(b, a)=\top$ implies $a=b$, for all $a, b \in U$.

We can now introduce the notions of fuzzy poset and fuzzy preposet as follows:

- An $\mathbb{L}-f u z z y$ poset is a pair $\mathbb{U}=\left(U, \rho_{U}\right)$ in which $\rho_{U}$ is a reflexive, antisymmetric and transitive $\mathbb{L}$-fuzzy relation on $U$.

- An $\mathbb{L}$-fuzzy preposet is a pair $\mathbb{U}=\left(U, \rho_{U}\right)$ in which $\rho_{U}$ is a reflexive and transitive $\mathbb{L}$-fuzzy relation on $U$.

We will need the following order-related notions in the fuzzy framework:

Let $\mathbb{U}=\left\langle U, \rho_{U}\right\rangle$ be a fuzzy poset.

(i) The crisp set of upper bounds of a fuzzy set $X$ on $\mathbb{U}$ is defined as

$$
\mathrm{Up}(X)=\left\{a \in U \mid X(u) \leq \rho_{A}(u, a), \text { for all } u \in U\right\} .
$$


(ii) The upset and downset of an element $a \in U$ are defined as fuzzy sets $a^{\uparrow}, a^{\downarrow}: U \rightarrow L$, where $a^{\downarrow}(u)=\rho_{U}(u, a)$ and $a^{\uparrow}(u)=\rho_{U}(a, u)$ for all $u \in U$.

(iii) An element $a \in U$ is called a maximum of a fuzzy set $X$ if $X(a)=\top$ and $X \subseteq a^{\downarrow}$. The definition of a minimum is similar.

In absence of antisymmetry it is possible that several maximum (resp. minimum) elements for $X$ exist, which will be called $p$-maximum (resp. p-minimum). We will write $\mathrm{p}$-max $X$ (resp. $\mathrm{p}-\min X)$ to denote the set of p-maxima (resp. pminima) of $X$.

Remark 1. Although uniqueness is lost, given two p-maximum (resp. pminimum) elements $x$ and $y$, we have that $\rho_{U}(x, y)=\top$. This property will be relevant later in subsequent sections.

We can now recall the extension to the fuzzy case provided by Yao and Lu, also used in [8], which can be stated as follows:

Definition 3 ([32]). Let $\mathbb{A}=\left\langle A, \rho_{A}\right\rangle$ and $\mathbb{B}=\left\langle B, \rho_{B}\right\rangle$ be fuzzy preposets. $A$ pair of mappings $f: A \rightarrow B$ and $g: B \rightarrow A$ forms a Galois connection between $\mathbb{A}$ and $\mathbb{B}$, denoted $(f, g): \mathbb{A} \leftrightharpoons \mathbb{B}$ if, for all $a \in A$ and $b \in B$, the equality $\rho_{A}(a, g(b))=\rho_{B}(f(a), b)$ holds.

Note that we have maintained the original term used by Yao and Lu, although it technically corresponds to an adjunction, not a Galois connection.

\section{When the Domain Has the Structure of Fuzzy Preposet}

In this section, we consider a mapping $f: \mathbb{A} \rightarrow B$ from a fuzzy preposet $\mathbb{A}=$ $\left\langle A, \rho_{A}\right\rangle$ into an unstructured set $B$, and characterize those situations in which $B$ can be endowed with a fuzzy preorder relation and an isotone mapping $g: B \rightarrow A$ can be built such that the pair $(f, g)$ is an adjunction.

Let $\mathbb{A}=\left\langle A, \rho_{A}\right\rangle$ be a fuzzy preposet, and consider a mapping $f: A \rightarrow B$. The fuzzy p-kernel relation $\cong_{A}$ is the $\otimes$-transitive closure of the union of the fuzzy equivalence relations $\approx_{A}$ and $\equiv_{f}$, where

$$
\left(a_{1} \approx_{A} a_{2}\right)=\rho_{A}\left(a_{1}, a_{2}\right) \otimes \rho_{A}\left(a_{2}, a_{1}\right) \quad \text { for all } a_{1}, a_{2} \in A .
$$

and

$$
\left(a_{1} \equiv_{f} a_{2}\right)= \begin{cases}\perp & \text { if } f\left(a_{1}\right) \neq f\left(a_{2}\right), \\ \top & \text { if } f\left(a_{1}\right)=f\left(a_{2}\right) .\end{cases}
$$

Note that $\cong_{A}$ is also a fuzzy equivalence relation and the fuzzy equivalence classes $[a]_{\cong_{A}}: A \rightarrow L$ are the fuzzy sets defined by

$$
[a]_{A}(x)=\left(x \cong_{A} a\right) .
$$

In the definition of the inherited structure, and also in the right adjoint, we will make use of (some of) the following fuzzy powerings:

Given $(A, \rho)$ and $X, Y \subseteq A$, we define the Hoare, Smyth and full fuzzy powerings as follows: 
1. $\rho_{H}(X, Y)=\bigwedge_{x \in X} \bigvee_{y \in Y} \rho(x, y)$

2. $\rho_{S}(X, Y)=\bigwedge_{y \in Y} \bigvee_{x \in X} \rho(x, y)$

3. $\rho_{\propto}(X, Y)=\bigwedge_{x \in X} \bigwedge_{y \in Y} \rho(x, y)$

We can now state necessary and sufficient conditions for the existence of a right adjoint from a fuzzy preposet to an unstructured set.

Theorem 1. Let $\mathbb{A}=\left\langle A, \rho_{A}\right\rangle$ be a fuzzy preposet, and consider a mapping $f: A \rightarrow B$, then there exist a fuzzy preorder relation $\rho_{B}$ on $B$ and a mapping $g: B \rightarrow A$ such that $(f, g): \mathbb{A} \leftrightharpoons \mathbb{B}$ if and only if there exists a subset $S \subseteq A$ such that, for all $a, a_{1}, a_{2} \in A$ :

1. $S \subseteq \bigcup_{a \in A} \mathrm{p}-\max [a] \cong_{A}$.

2. $\mathrm{p}-\min \left(\mathrm{Up}\left([a]_{\cong_{A}}\right) \cap S\right) \neq \varnothing$

3. $\rho_{A}\left(a_{1}, a_{2}\right) \leq \rho_{H}\left(\mathrm{p}-\min \left(\mathrm{Up}\left(\left[a_{1}\right]_{\cong_{A}}\right) \cap S\right), \mathrm{p}-\min \left(\mathrm{Up}\left(\left[a_{2}\right]_{\cong_{A}}\right) \cap S\right)\right)$.

The proof of the theorem is completely constructive, and the ordered structure on $B$ is given as follows:

For any $a_{0} \in A$, there exist a number of suitable definitions of $g: B \rightarrow A$, and all of them can be specified as follows:

- If $b \in f(A)$, then $g(b)$ is any element in $\mathrm{p}-\min \left(\mathrm{Up}\left(\left[x_{b}\right]_{\cong_{A}}\right) \cap S\right)$ for $x_{b} \in f^{-1}(b)$.

- If $b \notin f(A)$, then $g(b)$ is any element in $\mathrm{p}-\min \left(\mathrm{Up}\left(\left[a_{0}\right]_{\cong_{A}}\right) \cap S\right)$.

Finally, the fuzzy relation $\rho_{B}^{a_{0}}: B \times B \rightarrow L$ is defined as follows

$$
\rho_{B}^{a_{0}}\left(b_{1}, b_{2}\right)=\rho_{A}\left(g\left(b_{1}\right), g\left(b_{2}\right)\right) \text {. }
$$

\section{Changing Crisp Equality by a Fuzzy Equivalence Relation}

A further step towards generalization to the fuzzy realm is possible when considering fuzzy equivalence relations in each of the involved sets instead of the mere equality relation. This leads to a notion of fuzzy Galois connection in which the mappings $f$ and $g$ can be seen, in some sense, as fuzzy mappings instead of being crisp ones.

In this section, we consider the case where there are two underlying fuzzy equivalence relations in both the domain and the codomain of the mapping $f$, more specifically, $f$ is a morphism between the fuzzy structures $\left\langle A, \approx_{A}\right\rangle$ and $\left\langle B, \approx_{B}\right\rangle$ where, in addition, $\left\langle A, \approx_{A}\right\rangle$ is a fuzzy preordered structure.

The additional consideration of an underlying fuzzy equivalence relation suggests considering the following notions: 
(i) A fuzzy structure $\mathcal{A}=\left\langle A, \approx_{A}\right\rangle$ is a set $A$ endowed with a fuzzy equivalence relation $\approx_{A}$.

(ii) A morphism between two fuzzy structures $\mathcal{A}$ and $\mathcal{B}$ is a mapping $f: A \rightarrow B$ such that for all $a_{1}, a_{2} \in A$ the following inequality holds: $\left(a_{1} \approx_{A} a_{2}\right) \leq$ $\left(f\left(a_{1}\right) \approx_{B} f\left(a_{2}\right)\right)$. In this case, we write $f: \mathcal{A} \rightarrow \mathcal{B}$, and we say that $f$ is compatible with $\approx_{A}$ and $\approx_{B}$.

(iii) A morphism between two fuzzy structures $\mathcal{A}$ and $\mathcal{B}$ is said to be

$\approx$-injective if $\left(f\left(a_{1}\right) \approx_{B} f\left(a_{2}\right)\right) \leq\left(a_{1} \approx_{A} a_{2}\right)$, for all $a_{1}, a_{2} \in A$.

$\approx$-surjective if for all $b \in B$ there exists $a \in A$ such that $\left(f(a) \approx_{B} b\right)=\top$.

(iv) Let $\mathcal{B}=\left\langle B, \approx_{B}\right\rangle$ be a fuzzy structure, and consider a crisp subset $X \subseteq B$.

A mapping $h: B \rightarrow X$ is said to be a contraction if it is a morphism $h: \mathcal{B} \rightarrow\left\langle X, \approx_{B}\right\rangle$ and $h(x)=x$ for all $x \in X$.

Given a fuzzy structure $\mathcal{A}=\left\langle A, \approx_{A}\right\rangle$, we can now introduce the notion of fuzzy preordered structure as a pair $\mathbb{A}=\left\langle\mathcal{A}, \rho_{A}\right\rangle$ in which $\rho_{A}$ is a fuzzy relation that is $\approx_{A}$-reflexive, $\otimes-\approx_{A}$-antisymmetric and $\otimes$-transitive, where

(i) $\approx_{A}$-reflexive means $\left(a_{1} \approx_{A} a_{2}\right) \leq \rho_{A}\left(a_{1}, a_{2}\right)$ for all $a_{1}, a_{2} \in A$.

(ii) $\otimes-\approx_{A}$-antisymmetric means $\rho_{A}\left(a_{1}, a_{2}\right) \otimes \rho_{A}\left(a_{2}, a_{1}\right) \leq\left(a_{1} \approx_{A} a_{2}\right)$ for all $a_{1}, a_{2} \in A$.

If the underlying fuzzy structure is not clear from the context, we will sometimes write a fuzzy preordered structure as a triplet $\mathbb{A}=\left\langle A, \approx_{A}, \rho_{A}\right\rangle$.

The formal notion of p-maximum (resp. p-minimum) in the context of fuzzy preordered structures is exactly the same as in the previous section; however, the use of the underlying fuzzy equivalence relation leads to different properties. Observe that, given two p-maxima $x_{1}, x_{2}$ of a fuzzy set $X$ in a fuzzy preordered structure, we obtain $\rho_{A}\left(x_{1}, x_{2}\right)=\top=\rho_{A}\left(x_{2}, x_{1}\right)$ and by $\otimes-\approx_{A}$-antisymmetry, also $\left(x_{1} \approx_{A} x_{2}\right)=\top$.

A reasonable approach to introduce the notion of Galois connection between fuzzy preordered structures $\mathbb{A}$ and $\mathbb{B}$ would be the following:

Definition 4 ([9]). Let $\mathbb{A}$ and $\mathbb{B}$ be two fuzzy preordered structures. Given two morphisms $f: \mathcal{A} \rightarrow \mathcal{B}$ and $g: \mathcal{B} \rightarrow \mathcal{A}$, the pair $(f, g)$ is said to be a Galois connection between $\mathbb{A}$ and $\mathbb{B}$ (briefly, $(f, g): \mathbb{A} \leftrightharpoons \mathbb{B}$ ) if the following conditions hold for all $a, a_{1}, a_{2} \in A$ and $b, b_{1}, b_{2} \in B$ :

(G1) $\left(a_{1} \approx_{A} a_{2}\right) \otimes \rho_{A}\left(a_{2}, g(b)\right) \leq \rho_{B}\left(f\left(a_{1}\right), b\right)$

(G2) $\left(b_{1} \approx_{B} b_{2}\right) \otimes \rho_{B}\left(f(a), b_{1}\right) \leq \rho_{A}\left(a, g\left(b_{2}\right)\right)$

The previous definition behaves as expected, namely, it is equivalent to the standard equality for Galois connections. More specifically, the pair $(f, g)$ is a Galois connection between $\mathbb{A}$ and $\mathbb{B}$ if and only if both mappings are morphisms and $\rho_{A}(a, g(b))=\rho_{B}(f(a), b)$ for all $a \in A$ and $b \in B$.

Once again, we need the corresponding version of the kernel relation and its equivalence classes. These definitions are given below: 
Let $\mathcal{A}$ and $\mathcal{B}$ be two fuzzy structures and let $f: \mathcal{A} \rightarrow \mathcal{B}$ be a morphism. The fuzzy kernel relation $\equiv_{f}: A \times A \rightarrow L$ associated with $f$ is defined as follows, for $a_{1}, a_{2} \in A$,

$$
\left(a_{1} \equiv_{f} a_{2}\right)=\left(f\left(a_{1}\right) \approx_{B} f\left(a_{2}\right)\right) .
$$

The fuzzy kernel relation trivially is a fuzzy equivalence relation, and the equivalence class of an element $a \in A$ is the fuzzy set $[a]_{f}: A \rightarrow L$ defined by $[a]_{f}(u)=\left(f(a) \approx_{B} f(u)\right)$ for all $u \in A$.

Given a fuzzy preordered structure $\mathbb{A}=\left\langle A, \approx_{A}, \rho_{A}\right\rangle$, and crisp subsets $X, Y$ of $A$ and. The fuzzy relations $\approx_{A}$ and $\rho_{A}$ can be extended to the sets of pmaxima as follows:

$$
\begin{array}{r}
\left(\mathrm{p}-\max (X) \approx_{A} \mathrm{p}-\max (Y)\right) \stackrel{\text { def }}{=}\left(x \approx_{A} y\right) \\
\rho_{A}(\mathrm{p}-\max (X), \mathrm{p}-\max (Y)) \stackrel{\text { def }}{=} \rho_{A}(x, y)
\end{array}
$$

where $x$ (resp. $y$ ) can be any element in $\mathrm{p}-\max (X)(\operatorname{resp} . \mathrm{p}-\max (Y))$. It is not difficult to prove that the definition does not depend on the choice of $x$ and $y$.

The preceding notation allows us to state necessary conditions on $f$ in order to have a right adjoint in a more compact form which essentially follows the scheme already obtained in [8] and [19].

Theorem 2 (Necessary conditions). Consider two fuzzy preordered structures $\mathbb{A}$ and $\mathbb{B}$, together with two morphisms $f: \mathcal{A} \rightarrow \mathcal{B}$ and $g: \mathcal{B} \rightarrow \mathcal{A}$. If $(f, g)$ is a Galois connection between $\mathbb{A}$ and $\mathbb{B}$, then

1. $\mathrm{p}-\max \left([a]_{f}\right)$ is not empty for all $a \in A$.

2. $\rho_{A}\left(a_{1}, a_{2}\right) \leq \rho_{A}\left(\mathrm{p}-\max \left(\left[a_{1}\right]_{f}\right), \mathrm{p}-\max \left(\left[a_{2}\right]_{f}\right)\right)$, for all $a_{1}, a_{2} \in A$.

3. $\left(a_{1} \equiv_{f} a_{2}\right) \leq\left(\mathrm{p}-\max \left(\left[a_{1}\right]_{f}\right) \approx_{A} \mathrm{p}-\max \left(\left[a_{2}\right]_{f}\right)\right)$, for all $a_{1}, a_{2} \in A$.

We show now that the necessary conditions in Theorem 2 are sufficient in the case of $\mathrm{a} \approx$-surjective mapping.

Theorem 3 (Sufficient conditions). Consider a fuzzy preordered structure $\mathbb{A}$, a fuzzy structure $\mathcal{B}=\left\langle B, \approx_{B}\right\rangle$, and $a \approx$-surjective morphism $f: \mathcal{A} \rightarrow \mathcal{B}$. If the following conditions hold

1. $\mathrm{p}-\max \left([a]_{f}\right)$ is not empty for all $a \in A$;

2. $\rho_{A}\left(a_{1}, a_{2}\right) \leq \rho_{A}\left(\mathrm{p}-\max \left(\left[a_{1}\right]_{f}\right), \mathrm{p}-\max \left(\left[a_{2}\right]_{f}\right)\right)$, for all $a_{1}, a_{2} \in A$;

3. $\left(a_{1} \equiv_{f} a_{2}\right) \leq\left(\mathrm{p}-\max \left(\left[a_{1}\right]_{f}\right) \approx_{A} \mathrm{p}-\max \left(\left[a_{2}\right]_{f}\right)\right)$, for all $a_{1}, a_{2} \in A$;

then there exists $a \approx_{B}$-reflexive, $\otimes-\approx_{B}$-antisymmetric and $\otimes$-transitive fuzzy relation $\rho_{B}$ on $B$ and a morphism $g: \mathcal{B} \rightarrow \mathcal{A}$ such that $(f, g)$ is a Galois connection between the fuzzy preordered structures $\mathbb{A}$ and $\mathbb{B}=\left\langle\mathcal{B}, \rho_{B}\right\rangle$.

We also identify necessary and sufficient conditions in the case of a $\approx$-injective mapping.

Theorem 4. Consider two fuzzy preordered structures $\mathbb{A}=\left\langle\mathcal{A}, \rho_{A}\right\rangle$ and $\mathbb{B}=$ $\left\langle\mathcal{B}, \rho_{B}\right\rangle$. For $a \approx$-injective morphism $f: \mathcal{A} \rightarrow \mathcal{B}$, the following statements are equivalent: 
1. There exists a morphism $g: \mathcal{B} \rightarrow \mathcal{A}$ such that $(f, g): \mathbb{A} \leftrightharpoons \mathbb{B}$.

2. There exist a contraction $h:\left\langle B, \approx_{B}\right\rangle \rightarrow\left\langle f(A), \approx_{B}\right\rangle$ and a fuzzy relation $\rho_{f(A)}$ defined as $\rho_{f(A)}\left(f\left(a_{1}\right), f\left(a_{2}\right)\right)=\rho_{A}\left(a_{1}, a_{2}\right)$ such that the pair $(i, h)$ is a Galois connection between $\left\langle f(A), \approx_{B}, \rho_{f(A)}\right\rangle$ and $\left\langle B, \approx_{B}, \rho_{B}\right\rangle$, where $i: f(A) \rightarrow B$ denotes the canonical embedding.

The previous results lead to the systematic construction of the induced structure and the right adjoint in Algorithm 1.

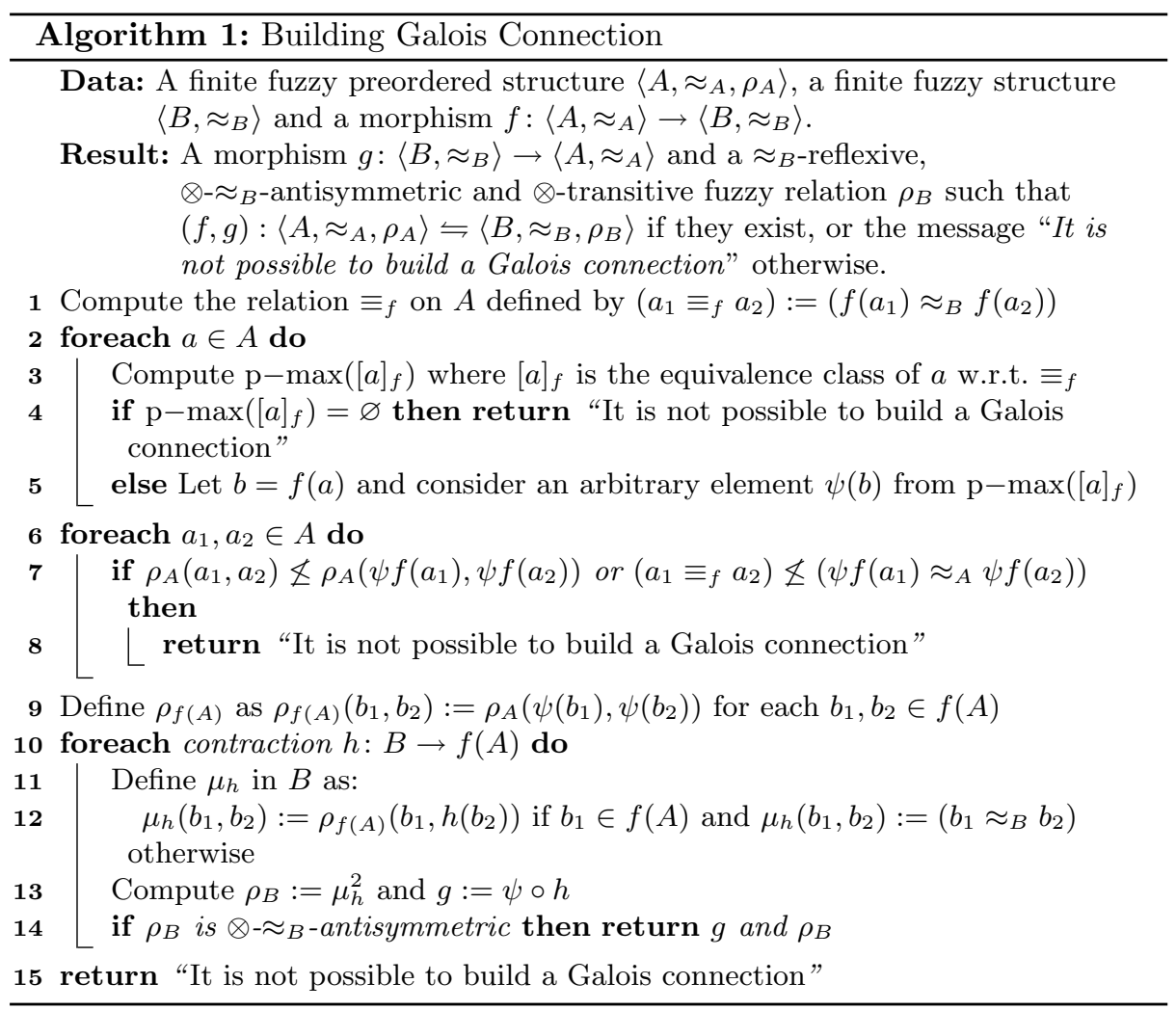

\section{Relational Galois Connections Between Fuzzy T-Digraphs}

We attempt here a first generalization of the notion of relational Galois connection to the fuzzy case. The focus is put on transitive fuzzy directed graphs, fuzzy T-digraphs for short, because of their interest for applications. One can find interesting theoretical applications of digraphs, for instance, Akram et al. [1] introduce the notion of fuzzy rough digraph and consider its application 
in decision making. In [2], Baykasoglu applies a fuzzy digraph model to quantify manufacturing flexibility. In [25], Koulouriotis and Ketipi develop a fuzzy digraph method for robot evaluation and selection, according to a given industrial application.

In this section, we focus specifically on providing an adequate notion of relational Galois connection between fuzzy T-digraphs which inherits most of the interesting equivalent characterizations of the notion of crisp Galois connection.

Our framework in this work is relational at the level of Galois connections (namely, the components of a Galois connection are crisp binary relations instead of functions) and fuzzy at the level of their domain and codomain.

We will use the following standard notions about relations: Given a binary relation $\mathcal{R} \subseteq A \times B$, the afterset $a^{\mathcal{R}}$ of an element $a \in A$ is defined as $\{b \in B \mid$ $a \mathcal{R} b\}$.

Definition 5. A pair $\mathbb{A}=(A, \rho)$ is said to be a fuzzy T-digraph if $\rho$ is a $\otimes$ transitive fuzzy relation on $A$.

The usual requirement that in a Galois condition both components should be antitone and their compositions inflationary leads to a preliminary approach to the definition of a relational Galois connection for fuzzy preposets.

Let us, firstly, fix the notions of antitone and inflationary relation in a fuzzy setting. Given $(A, \rho)$ and $(B, \rho)$ :

1. A relation $\mathcal{R} \subseteq A \times B$ is antitone if $\rho\left(a_{1}, a_{2}\right) \leq \rho\left(b_{2}, b_{1}\right)$ for all $b_{1} \in a_{1}^{\mathcal{R}}$ and $b_{2} \in a_{2}^{\mathcal{R}}$, or equivalently, $\rho\left(a_{1}, a_{2}\right) \leq \rho_{\propto}\left(a_{2}^{\mathcal{R}}, a_{1}^{\mathcal{R}}\right)$.

2. A relation $\mathcal{R} \subseteq A \times A$ is inflationary if $\rho\left(a_{1}, a_{2}\right)=\top$ for all $a_{2} \in a_{1}^{\mathcal{R}}$ or, equivalently, $\rho_{\propto}\left(a, a^{\mathcal{R}}\right)=\top$.

We can obtain the following proposition which links the properties of antitone and inflationary to a pair of inequalities with a certain flavour to Galois condition.

Proposition 1. Let $(A, \rho)$ and $(B, \rho)$ be fuzzy preposets and $\mathcal{R} \subseteq A \times B$ and $\mathcal{S} \subseteq B \times A$ be relations. Then $\mathcal{R}$ and $\mathcal{S}$ are antitone and $\mathcal{R} \circ \mathcal{S}$ and $\mathcal{S} \circ \mathcal{R}$ are inflationary if and only if the following inequalities hold:

$$
\rho_{H}\left(a, b^{\mathcal{S}}\right) \leq \rho_{S}\left(b, a^{\mathcal{R}}\right) \quad \text { and } \quad \rho_{H}\left(b, a^{\mathcal{R}}\right) \leq \rho_{S}\left(a, b^{\mathcal{S}}\right) .
$$

This proposition suggests to consider inequalities (2) as a tentative definition of relational Galois connection between fuzzy T-digraphs. To begin with, we have the following result.

Proposition 2. Let $(A, \rho)$ and $(B, \rho)$ be fuzzy T-digraphs and $\mathcal{R} \subseteq A \times B$ and $\mathcal{S} \subseteq B \times A$ be relations. If $\mathcal{R}$ and $\mathcal{S}$ are antitone and $\mathcal{R} \circ \mathcal{S}$ and $\mathcal{S} \circ \mathcal{R}$ are inflationary, then $(\mathcal{R}, \mathcal{S})$ satisfy condition $(2)$.

However, the following example shows that the converse does not hold.

Example 1. Consider the following fuzzy T-digraphs $\mathbb{A}=\left(\left\{a_{1}, a_{2}, a_{3}\right\}, \rho\right)$ and $\mathbb{B}=\left(\left\{b_{1}, b_{2}, b_{3}\right\}, \rho\right)$, and the relations $\mathcal{R} \subseteq A \times B$ and $\mathcal{S} \subseteq B \times A$ defined below: 


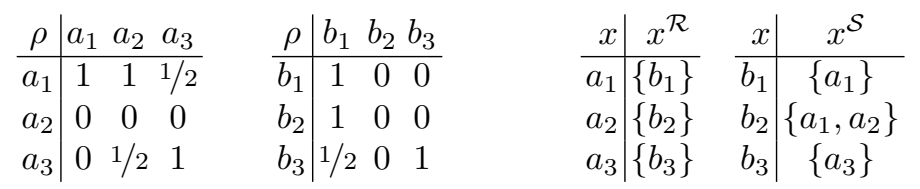

It is routine to check that $(\mathcal{R}, \mathcal{S})$ satisfies condition $(2)$. Nevertheless, $\mathcal{R} \circ \mathcal{S}$ is not inflationary, because $\left\{a_{1}\right\} \in a_{2}^{\mathcal{R} \circ \mathcal{S}}$, while $\rho\left(a_{2}, a_{1}\right)=0$ and $\rho_{S}\left(a_{2}, a_{2}^{\mathcal{R} \circ \mathcal{S}}\right)=$ $\rho_{S}\left(a_{1},\left\{a_{1}, a_{2}\right\}\right)=0$.

The question now is to discover some "missing" requirement which should be required in order to prove the converse of Proposition 2. Surprisingly, this requirement already appeared in previous sections as a property of the sets of pminima or p-maxima; namely, all the elements in the aftersets should be related with degree $T$. Formally, we have the following definition:

Definition 6. Let $(A, \rho)$ be a fuzzy $T$-digraph and $X \subseteq A$. We say that a nonempty set $X$ is a clique if for all $x, y \in X$ it holds $\rho(x, y)=\top$ or, equivalently, $\rho_{\propto}(X, X)=\top$.

Notice that given a fuzzy T-digraph $(A, \rho), X \subseteq A$ and $a \in A$, then if $X$ is a clique, we have that $\rho_{S}(a, X)=\rho_{\propto}(a, X)=\rho_{H}(a, X)$. As a result, the inequalities in (2) collapse into the equality $\rho_{\propto}\left(a, b^{\mathcal{S}}\right)=\rho_{\propto}\left(b, a^{\mathcal{R}}\right)$ and, furthermore, the following characterisation can be proved:

Theorem 5. Let $(A, \rho)$ and $(B, \rho)$ be fuzzy $T$-digraphs. Given $\mathcal{R} \subseteq A \times B$ and $\mathcal{S} \subseteq B \times A$ then, $\mathcal{R}$ and $\mathcal{S}$ are antitone and $\mathcal{R} \circ \mathcal{S}$ and $\mathcal{S} \circ \mathcal{R}$ are inflationary between $(A, \rho)$ and $(B, \rho)$ if and only if the following conditions hold:

(i) $\rho_{\propto}\left(a, b^{\mathcal{S}}\right)=\rho_{\propto}\left(b, a^{\mathcal{R}}\right)$ for all $a \in A$ and $b \in B$,

(ii) $a^{\mathcal{R}}$ and $b^{\mathcal{S}}$ are cliques for all $a \in A$ and $b \in B$.

As a consequence, we can give an adequate definition of relational Galois connection between fuzzy T-digraphs which, on the one hand, generalizes the Galois condition and, on the other hand, guarantees the properties of the components of the connection:

Definition 7. Let $(A, \rho)$ and $(B, \rho)$ be fuzzy T-digraphs and $\mathcal{R} \subseteq A \times B$ and $\mathcal{S} \subseteq B \times A$ be relations. We say that the pair $(\mathcal{R}, \mathcal{S})$ is a relational Galois connection if the following conditions hold:

(i) $\rho_{\propto}\left(a, b^{\mathcal{S}}\right)=\rho_{\propto}\left(b, a^{\mathcal{R}}\right)$ for all $a \in A$ and $b \in B$,

(ii) $a^{\mathcal{R}}$ and $b^{\mathcal{S}}$ are cliques for all $a \in A$ and $b \in B$.

\section{Conclusions and Future Work}

There are a number of possible options to extend the notion of a Galois connection to a fuzzy setting. We have surveyed some of the previous works in this area, and provided a somewhat unified presentation. In some cases, we have given a 
characterization theorem of the existence of a right adjoint for a given function. Moreover, we have provided the adequate notion of Galois connection between fuzzy T-digraphs, whilst the explicit construction of a right adjoint for a given relation is left for future work.

The relational generalization to fuzzy T-digraphs paves the way towards obtaining an operative notion of fuzzy relational Galois connection between fuzzy T-digraphs, and initiates the search for a characterization of the existence of a residual to a given fuzzy relation. On the other hand, it might enable a new approach to Formal Concept Analysis, provided that the definition of relational Galois connection is suitably adapted to formal contexts.

Acknowledgments. Partially supported by the Spanish Ministry of Science, Innovation, and Universities (MCIU), the State Agency of Research (AEI) and the European Social Fund (FEDER) through projects PGC2018-095869-B-I00 and TIN2017-89023-P, and Junta de Andalucía project UMA2018-FEDERJA-001.

\section{References}

1. Akram, M., Shumaiza, Arshad, M.: A new approach based on fuzzy rough digraphs for decision-making. J. Intell. Fuzzy Syst. 35(2), 2105-2121 (2018)

2. Baykasoglu, A.: A practical fuzzy digraph model for modeling manufacturing flexibility. Cybern. Syst. 40(6), 475-489 (2009)

3. Bělohlávek, R.: Fuzzy Galois connections. Math. Logic Q. 45(4), 497-504 (1999)

4. Bělohlávek, R., Osička, P.: Triadic fuzzy Galois connections as ordinary connections. Fuzzy Sets Syst. 249, 83-99 (2014)

5. Bloch, I.: Fuzzy sets for image processing and understanding. Fuzzy Sets Syst. 281, 280-291 (2015)

6. Brattka, V.: A Galois connection between turing jumps and limits. Log. Methods Comput. Sci. 14(3:13) (2018)

7. Butka, P., Pócs, J., Pócsová, J.: Isotone Galois connections and generalized onesided concept lattices. In: Choroś, K., Kopel, M., Kukla, E., Siemiński, A. (eds.) MISSI 2018. AISC, vol. 833, pp. 151-160. Springer, Cham (2019). https://doi.org/ 10.1007/978-3-319-98678-4_17

8. Cabrera, I., Cordero, P., Garcia-Pardo, F., Ojeda-Aciego, M., De Baets, B.: On the construction of adjunctions between a fuzzy preposet and an unstructured set. Fuzzy Sets Syst. 320, 81-92 (2017)

9. Cabrera, I., Cordero, P., Garcia-Pardo, F., Ojeda-Aciego, M., De Baets, B.: Galois connections between a fuzzy preordered structure and a general fuzzy structure. IEEE Trans. Fuzzy Syst. 26(3), 1274-1287 (2018)

10. Cabrera, I., Cordero, P., Muñoz-Velasco, E., Ojeda-Aciego, M., De Baets, B.: Relational Galois connections between transitive fuzzy digraphs. Math. Methods Appl. Sci. 43(9), 5673-5680 (2020)

11. Cabrera, I., Cordero, P., Ojeda-Aciego, M.: Galois connections in computational intelligence: a short survey. In: IEEE Symposium Series on Computational Intelligence (SSCI) (2017)

12. Cornelis, C., Medina, J., Verbiest, N.: Multi-adjoint fuzzy rough sets: definition, properties and attribute selection. Int. J. Approx. Reason. 55(1), 412-426 (2014) 
13. Denniston, J.T., Melton, A., Rodabaugh, S.E.: Formal contexts, formal concept analysis, and Galois connections. Electr. Proc. Theor. Comput. Sci. 129, 105-120 (2013)

14. Djouadi, Y., Prade, H.: Interval-valued fuzzy Galois connections: algebraic requirements and concept lattice construction. Fundamenta Informaticae 99(2), 169-186 (2010)

15. Dzik, W., Järvinen, J., Kondo, M.: Representing expansions of bounded distributive lattices with Galois connections in terms of rough sets. Int. J. Approx. Reason. 55(1), 427-435 (2014)

16. Díaz, J., Medina, J., Ojeda-Aciego, M.: On basic conditions to generate multiadjoint concept lattices via Galois connections. Int. J. Gen. Syst. 43(2), 149-161 (2014)

17. Faul, P.F.: Adjunctions in the study of broadcast domination with a cost function. Aust. J. Comb. 72, 70-81 (2018)

18. Frascella, A.: Fuzzy Galois connections under weak conditions. Fuzzy Sets Syst. 172(1), 33-50 (2011)

19. García-Pardo, F., Cabrera, I., Cordero, P., Ojeda-Aciego, M., Rodríguez, F.: On the definition of suitable orderings to generate adjunctions over an unstructured codomain. Inf. Sci. 286, 173-187 (2014)

20. Georgescu, G., Popescu, A.: Non-commutative fuzzy Galois connections. Soft Comput. 7(7), 458-467 (2003)

21. Gibbons, J., Henglein, F., Hinze, R., Wu, N.: Relational algebra by way of adjunctions. Proc. ACM Program. Lang. 2, 86:1-86:28 (2018)

22. González-Hidalgo, M., Massanet, S., Mir, A., Ruiz-Aguilera, D.: A fuzzy morphological hit-or-miss transform for grey-level images: a new approach. Fuzzy Sets Syst. 286, 30-65 (2016)

23. Gutiérrez-García, J., Mardones-Pérez, I., de Prada-Vicente, M.A., Zhang, D.: Fuzzy Galois connections categorically. Math. Log. Q. 56(2), 131-147 (2010)

24. Konecny, J.: Isotone fuzzy Galois connections with hedges. Inf. Sci. 181, 1804-1817 (2011)

25. Koulouriotis, D.E., Ketipi, M.K.: A fuzzy digraph method for robot evaluation and selection. Expert Syst. Appl. 38(9), 11901-11910 (2011)

26. Kycia, R.: Landauer's principle as a special case of Galois connection. Entropy 20(12), 971 (2018)

27. Moraschini, T.: A logical and algebraic characterization of adjunctions between generalized quasi-varieties. J. Symb. Log. 83(3), 899-919 (2018)

28. Ore, Ø.: Galois connexions. Trans. Am. Math. Soc. 55, 493-513 (1944)

29. Shi, Y., Nachtegael, M., Ruan, D., Kerre, E.: Fuzzy adjunctions and fuzzy morphological operations based on implications. Int. J. Intell. Syst. 24(12), 1280-1296 (2009)

30. Sussner, P.: Lattice fuzzy transforms from the perspective of mathematical morphology. Fuzzy Sets Syst. 288, 115-128 (2016)

31. Yao, W., Han, S., Wang, R.: Lattice-theoretic contexts and their concept lattices via Galois ideals. Inf. Sci. 339, 1-18 (2016)

32. Yao, W., Lu, L.-X.: Fuzzy Galois connections on fuzzy posets. Math. Log. Q. 55(1), 105-112 (2009)

33. Yao, Y.: Rough-set concept analysis: interpreting RS-definable concepts based on ideas from formal concept analysis. Inf. Sci. 346-347, 442-462 (2016) 